\title{
LEVI-PARALLEL HYPERSURFACES IN A COMPLEX SPACE FORM
}

By

Jong Taek CHO

\begin{abstract}
In this paper, we classify a Hopf hypersurface in a nonflat complex space form whose Levi-form is parallel with respect to the generalized Tanaka-Webster connection.
\end{abstract}

\section{Introduction}

Let $\tilde{M}=\left(\tilde{M}^{n}, J, \tilde{g}\right)$ be a complex $n$-dimensional Kählerian manifold with complex structure $J$ and Kählerian metric $\tilde{g}$. Let $M$ be an oriented real hypersurface in $\tilde{M}, g$ be the induced metric and $\eta$ be the 1 -form defined by $\eta(X)=g(X, \xi)$ where $\xi=-J N$ and $N$ is a unit normal vector field on $M$. Then $M$ has an (integrable) CR-structure associated with the complex structure of the ambient space. Let $T M$ be the tangent bundle of $M$ and $D$ be the subbundle of $T M$ (or the $(2 n-2)$-dimensional distribution) which is defined by $\eta=0$. We denote by $\mathbf{C} D=D \otimes \mathbf{C}$ its complexification. Then we see that $D$ is holomorphic (or maximally invariant by $J$ ) and

$$
\mathscr{H}=\{X-i J X: X \in D\}
$$

defines an $C R$-structure on $M$. That is, $\mathscr{H}$ satisfies the following properties:

(i) each fiber $\mathscr{H}_{x}(x \in M)$ is of complex dimension $n-1$,

(ii) $\mathscr{H} \cap \overline{\mathscr{H}}=\{0\}$,

(iii) $[\mathscr{H}, \mathscr{H}] \subset \mathscr{H}$ (integrability).

Furthermore, we have $\mathbf{C} D=\mathscr{H} \oplus \overline{\mathscr{H}}$. We call $\{D, J\}$ the real representation of $\mathscr{H}$. Then for $\{D, J\}$ we define the Levi form by

$$
L: D \times D \rightarrow \mathscr{F}(M), \quad L(X, Y)=d \eta(X, J Y)
$$

\section{Mathematics Subject Classification (2000): 53C15, 53C40, 53D15.}

This study was financially supported by research fund of Chonnam National University in 2003. Key words and phrases. Tanaka-Webster connection, Levi parallel hypersurfaces, complex space forms.

Received February 2, 2005. 
where $\mathscr{F}(M)$ denotes the algebra of differentiable functions on $M$. If the Levi form is hermitian, then the CR-structure is called pseudo-hermitian, in addition, in the case that the Levi form is non-degenerate (positive or negative definite, resp.), then the $\mathrm{CR}$ structure is called a non-degenerate (strongly pseudo-convex, resp.) pseudo-hermitian CR structure. Recently, Y. T. Siu [14] proved the nonexistence of compact smooth Levi-flat hypersurfaces in complex projective spaces of dimension $\geq 3$. Here, it is remarkable that the assumption of compactness in Siu's theorem has a crucial role. Actually, there are non-complete examples which are Levi-flat in a complex projective space (see section 2). Anyway, the examples of Levi-flat hypersurfaces which are known so far are not Hopf. In this situation, we prove that there does not exist a Levi-flat Hopf hypersurface (Theorem 3).

On the other hand, the Tanaka-Webster connection ([19], [20]) is defined as a canonical affine connection on a pseudo-hermitian, non-degenerate, integrable CR manifold. For contact metric manifolds, their associated almost CR structures are pseudo-hermitian and strongly pseudo-convex, but they are not in general integrable. For a non-zero real number $k$, the author [7] defined the generalized Tanaka-Webster connection (in short, the g.-Tanaka-Webster connection) $\hat{\nabla}$ for real hypersurfaces in Kählerian manifolds. The g.-Tanaka-Webster connection $\hat{\nabla}$ coincides with the Tanaka-Webster connection if real hypersurfaces satisfy $\phi A+A \phi=2 k \phi$ (Proposition 2). The covariant differentiation of the Levi form $L$ with respect to the g.-Tanaka-Webster connection $\hat{\nabla}$ is well-defined:

$$
\left(\hat{\nabla}_{X} L\right)(Y, Z)=X L(Y, Z)-L\left(\hat{\nabla}_{X} Y, Z\right)-L\left(Y, \hat{\nabla}_{X} Z\right)
$$

for any $X, Y, Z \in D$. Then we say that $M$ is Levi-parallel with respect to the g.-Tanaka-Webster connection or shortly Levi-parallel if $M$ satisfies

$$
g\left(\left(\hat{\nabla}_{X} L\right)(Y, Z)\right)=0
$$

for any vector fields $X, Y, Z \in D$. We note that a Levi-flat hypersurface is Leviparallel (see (2) in Remark 1).

A complex $n$-dimensional complete and simply connected Kählerian manifold of constant holomorphic sectional curvature $c$ is called a complex space form, which is denoted by $\tilde{M}_{n}(c)$. A complex space form consists of a complex projective space $P_{n} \mathbf{C}$, a complex Euclidean space $E_{n} \mathbf{C}$ or a complex hyperbolic space $H_{n} \mathbf{C}$, according as $c>0, c=0$ or $c<0$. R. Takagi $[16,17]$ classified the homogeneous real hypersurfaces of $P_{n} \mathrm{C}$ into six types. T. E. Cecil and P. J. Ryan [6] extensively studied a real hypersurface whose structure vector $\xi$ is a principal curvature vector, which is realized as tubes over certain submanifolds in $P_{n} \mathbf{C}$, by using its focal map. A real hypersurface of a complex space form is said to 
be a Hopf hypersurface if its structure vector is a principal curvature vector. By making use of those results and the mentioned work of R. Takagi, M. Kimura [9] proved the local classification theorem for Hopf hypersurfaces of $P_{n} \mathrm{C}$ whose all principal curvatures are constant. For the case $H_{n} \mathbf{C}$, J. Berndt [3] proved the classification theorem for Hopf hypersurfaces whose all principal curvatures are constant.

The main purpose of the present paper is to classify real hypersurfaces of $\tilde{M}_{n}(c), c \neq 0$ whose Levi form is parallel with respect to the generalized TanakaWebster connection. More specifically, in section 4, we prove

MAIN THEOREM. Let $M$ be a Hopf hypersurface of a complex space form $\tilde{M}_{n}(c), c \neq 0$. Suppose that $M$ is Levi-parallel with respect to the g.-TanakaWebster connection. Then we have the following.

(I) If $\tilde{M}_{n}(c)=P_{n} \mathbf{C}$, then $M$ is locally congruent to one of:

$\left(A_{1}\right)$ a geodesic hypersphere of radius $r$, where $0<r<\frac{\pi}{2}$,

$\left(A_{2}\right)$ a tube of radius $r$ over a totally geodesic $P_{k} \mathbf{C}(1 \leq k \leq n-2)$, where $0<r<\frac{\pi}{2}$

(B) a tube of radius $r$ over a complex quadric $Q_{n-1}$, where $0<r<\frac{\pi}{4}$.

(II) If $\tilde{M}_{n}(c)=H_{n} \mathbf{C}$, then $M$ is locally congruent to one of:

$\left(A_{0}\right)$ a horosphere,

$\left(A_{1}\right)$ a geodesic hypersphere or a tube over a complex hyperbolic hyperplane $H_{n-1} \mathbf{C}$,

$\left(A_{2}\right)$ a tube over a totally geodesic $H_{k} \mathbf{C}(1 \leq k \leq n-2)$,

(B) a tube over a totally real hyperbolic space $H_{n} \mathbf{R}$.

\section{The Generalized Tanaka-Webster Connection for Real Hypersurfaces}

In this paper, all manifolds are assumed to be connected and of class $C^{\infty}$ and the real hypersurfaces are supposed to be oriented. First, we give a brief review of several fundamental concepts and formulas on almost contact structure. An odddimensional smooth manifold $M^{2 n+1}$ has an almost contact structure if it admits a vector $\xi$, a 1 -form $\eta$ and a (1,1)-tensor field $\varphi$ satisfying

$$
\eta(\xi)=1 \quad \text { and } \quad \varphi^{2} X=-X+\eta(X) \xi .
$$

Then there exists a compatible Riemannian metric $g$ :

$$
g(\varphi X, \varphi Y)=g(X, Y)-\eta(X) \eta(Y)
$$

for all vector fields $X$ and $Y$ on $M$. We call $(\eta, \xi, \varphi, g)$ an almost contact metric structure of $M$ and $M=(M ; \eta, \xi, \varphi, g)$ an almost contact metric manifold. For 
an almost contact metric manifold $M$ we define its fundamental 2-form $\Phi$ by $\Phi(X, Y)=g(\varphi X, Y)$. If

$$
\Phi=d \eta
$$

$M$ is called a contact metric manifold. We refer to [4] on contact metric geometry for more detail.

For an almost contact metric manifold $M$, the tangent space $T_{p} M$ of $M$ at each point $p \in M$ is decomposed as $T_{p} M=D_{p} \oplus\{\xi\}_{p}$ (direct sum), where we denote $D_{p}=\left\{v \in T_{p} M \mid \eta(v)=0\right\}$. Then $D: p \rightarrow D_{p}$ defines a distribution orthogonal to $\xi$. The restriction $\bar{\varphi}=\varphi \mid D$ of $\varphi$ to $D$ defines an almost complex structure to $D$. If the associated Levi form $L$, defined by

$$
L(X, Y)=d \eta(X, \bar{\varphi} Y),
$$

$X, Y \in D$, is hermitian, then $(\eta, \bar{\varphi})$ is called a pseudo-hermitian $\mathrm{CR}$ structure and in addition, if its Levi form is non-degenerate (positive or negative definite, resp.), then $(\eta, \bar{\varphi})$ is called a non-degenerate (strongly pseudo-convex, resp.) pseudohermitian CR structure. Moreover, if the following conditions are satisfied:

$$
[\bar{\varphi} X, \bar{\varphi} Y]-[X, Y] \in D
$$

and

$$
[\bar{\varphi}, \bar{\varphi}](X, Y)=0
$$

for all $X, Y \in D$, where $[\bar{\varphi}, \bar{\varphi}]$ is the Nijenhuis torsion of $\bar{\varphi}$, then the pair $(\eta, \bar{\varphi})$ is called a pseudo-hermitian, non-degenerate, (strongly pseudo-convex, resp.) integrable CR structure associated with the almost contact metric structure $(\eta, \xi, \varphi, g)$. In particular, for a contact metric manifold its associated CR structure is pseudo-hermitian, strongly pseudo-convex but is not in general integrable. For further details about CR structures, we refer for example to [2], [5], [18].

Let $M$ be a real hypersurface of a Kählerian manifold $\tilde{M}=(\tilde{M} ; J, \tilde{g})$ and $N$ a global unit normal vector on $M$. By $\tilde{\nabla}, A$ we denote the Levi-Civita connection in $\tilde{M}$ and the shape operator with respect to $N$, respectively. Then the Gauss and Weingarten formulas are given respectively by

$$
\tilde{\nabla}_{X} Y=\nabla_{X} Y+g(A X, Y) N, \quad \tilde{\nabla}_{X} N=-A X
$$

for any vector fields $X$ and $Y$ tangent to $M$, where $g$ denotes the Riemannian metric of $M$ induced from $\tilde{g}$. An eigenvector (resp. eigenvalue) of the shape operator $A$ is called a principal curvature vector (resp. principal curvature). For any vector field $X$ tangent to $M$, we put 


$$
J X=\varphi X+\eta(X) N, \quad J N=-\xi .
$$

We easily see that the structure $(\eta, \xi, \varphi, g)$ is an almost contact metric structure on $M$. From the condition $\tilde{\nabla} J=0$, the relations (2.1) and by making use of the Gauss and Weingarten formulas, we have

$$
\begin{gathered}
\left(\nabla_{X} \varphi\right) Y=\eta(Y) A X-g(A X, Y) \xi, \\
\nabla_{X} \xi=\varphi A X .
\end{gathered}
$$

By using (2.2) and (2.3), we see that a real hypersurface in a Kählerian manifold always satisfies (1.2) and (1.3), the integrability condition of the associated almost CR structure. From (1.1) and (2.3) we have

Proposition 1. Let $M=(M ; \eta, \xi, \varphi, g)$ be a real hypersurface of a Kählerian manifold. The almost contact metric structure of $M$ is contact metric if and only if $\varphi A+A \varphi= \pm 2 \varphi$, where \pm is determined by the orientation.

The Tanaka-Webster connection ([19], [20]) is the canonical affine connection defined in a non-degenerate integrable CR manifold. Tanno ([18]) defined the generalized Tanaka-Webster connection for contact metric manifolds by the canonical connection which coincides with the Tanaka-Webster connection if the associated almost CR structure is integrable. We define the generalized TanakaWebster connection (in short, the g.-Tanaka-Webster connection) for real hypersurfaces of Kählerian manifolds by the naturally extended one of Tanno's generalized Tanaka-Webster connection for contact metric manifolds.

We recall Tanno's generalized Tanaka-Webster connection $\hat{\nabla}$ for contact metric manifolds:

$$
\hat{\nabla}_{X} Y=\nabla_{X} Y+\left(\nabla_{X} \eta\right)(Y) \xi-\eta(Y) \nabla_{X} \xi-\eta(X) \varphi Y
$$

for all vector fields $X$ and $Y$.

Taking account of (2.3), the g.-Tanaka-Webster connection for real hypersurfaces of Kählerian manifolds, which is denoted by the same symbol $\hat{\nabla}$ as the one for contact metric manifolds, is naturally defined by (cf. [7])

$$
\hat{\nabla}_{X} Y=\nabla_{X} Y+g(\varphi A X, Y) \xi-\eta(Y) \varphi A X-k \eta(X) \varphi Y,
$$

where $k$ is a non-zero real number. We put $F_{X} Y=g(\varphi A X, Y) \xi-\eta(Y) \varphi A X-$ $k \eta(X) \varphi Y$. Then the torsion tensor $\hat{T}$ is given by $\hat{T}(X, Y)=F_{X} Y-F_{Y} X$. Also, by using (1.2), (1.3), (2.2), (2.3) and (2.4) we can see that

$$
\hat{\nabla} \eta=0, \quad \hat{\nabla} \xi=0, \quad \hat{\nabla} g=0, \quad \hat{\nabla} \varphi=0 .
$$


and

$$
\hat{T}(X, Y)=2 d \eta(X, Y) \xi, \quad X, Y \in D .
$$

We note that the associated Levi form is

$$
L(X, Y)=\frac{1}{2} g((\bar{\varphi} \bar{A}+\bar{A} \bar{\varphi}) X, \bar{\varphi} Y)
$$

where we denote by $\bar{A}$ the restriction $A$ to $D$. If $M$ satisfies $\varphi A+A \varphi=2 k \varphi$, then we see that the associated $\mathrm{CR}$ structure is pseudo-hermitian, strongly pseudoconvex and further satisfies $\hat{T}(\xi, \varphi Y)=-\varphi \hat{T}(\xi, Y)$, and hence the generalized Tanaka-Webster connection $\hat{\nabla}$ coincides with the Tanaka-Webster connection. Namely, we have (cf. [7])

Proposition 2. Let $M=(M ; \eta, \xi, \varphi, g)$ be a real hypersurface of a Kählerian manifold. If $M$ satisfies $\varphi A+A \varphi=2 k \varphi$, then the associated CR-structure is pseudo-hermitian, strongly pseudo-convex, integrable, and further the generalized Tanaka-Webster connection $\hat{\nabla}$ coincides with the Tanaka-Webster connection.

Since the structure vector field $\xi$ is $\hat{\nabla}$-parallel, we see that $\hat{\nabla}_{X} Y$ for $X, Y \in D$ still belongs to $D$. We define the covariant differentiation of the Levi form $L$ with respect to the g.-Tanaka-Webster connection $\hat{\nabla}$ as follows:

$$
\left(\hat{\nabla}_{X} L\right)(Y, Z)=X L(Y, Z)-L\left(\hat{\nabla}_{X} Y, Z\right)-L\left(Y, \hat{\nabla}_{X} Z\right)
$$

for any $X, Y, Z \in D$.

\section{Real Hypersurfaces of a Complex Space Form}

Let $\tilde{M}=\tilde{M}_{n}(c)$ be a non-flat complex space form of constant holomorphic sectional curvature $c(\neq 0)$ and let $M$ a real hypersurface of $\tilde{M}$. Then we have the following Gauss and Codazzi equations:

$$
\begin{aligned}
R(X, Y) Z= & \frac{c}{4}\{g(Y, Z) X-g(X, Z) Y \\
& +g(\varphi Y, Z) \varphi X-g(\varphi X, Z) \varphi Y-2 g(\varphi X, Y) \varphi Z\} \\
& +g(A Y, Z) A X-g(A X, Z) A Y \\
\left(\nabla_{X} A\right) Y- & \left(\nabla_{Y} A\right) X=\frac{c}{4}\{\eta(X) \varphi Y-\eta(Y) \varphi X-2 g(\varphi X, Y) \xi\}
\end{aligned}
$$


for any tangent vector fields $X, Y, Z$ on $M$. We now suppose that $M$ is a Hopf hypersurface, that is, $A \xi=\alpha \xi$. Then we already know that $\alpha$ is constant (see [8]). Differentiating this covariantly along $M$, and then by using (2.3) we have

$$
\left(\nabla_{X} A\right) \xi=\alpha \varphi A X-A \varphi A X,
$$

and further by using (3.2) we obtain

$$
\left(\nabla_{\xi} A\right) X=\frac{c}{4} \varphi X+\alpha \varphi A X-A \varphi A X
$$

for any vector field $X$ on $M$. From this, we have

$$
2 A \varphi A X-\frac{c}{2} \varphi X=\alpha(\varphi A+A \varphi) X .
$$

Here, we assume that $A X=\lambda X$ for a unit vector field $X$ orthogonal to $\xi$, then

$$
(2 \lambda-\alpha) A \varphi X=\left(\alpha \lambda+\frac{c}{2}\right) \varphi X
$$

Now, we prove

THEOREM 3. There does not exist a Levi-flat Hopf hypersurface in a non-flat complex space form.

Proof. Suppose that $M$ is Hopf and Levi-flat. Then $A \xi=\alpha \xi$ and we get

$$
\varphi A X+A \varphi X=0
$$

for any $X \in D$. We assume $A X=\lambda X$. Since $\xi$ is a principal curvature vector by using (3.3) we have $2 \lambda^{2}+\frac{c}{2}=0$, which shows $c<0$. Then we see that $M$ has at most three constant principal curvatures $\lambda, \mu$ and $\alpha$, and further we see that $\mu=-\lambda$. But, Corollary 1 in [3] states that $\lambda \mu+c / 4=0$. Thus, we have a contradiction.

We remark here that there are examples of Levi-flat hypersurfaces which are not Hopf. We say that $M$ is a ruled real hypersurface of $\tilde{M}_{n}(c), c \neq 0$ if there is a foliation of $M$ by complex hyperplanes $\tilde{M}_{n-1}(c)$. In other words, $M$ is ruled if and only if $D$ is integrable and its integral manifold is a totally geodesic sub- 
manifold $\tilde{M}_{n-1}(c)$. Then we easily see that a ruled real hypersurface is Levi-flat. In fact, its shape operator may be written down as following:

$$
\begin{gathered}
A \xi=\alpha \xi+\mu U \quad(\mu \neq 0), \\
A U=\mu \xi, \\
A Z=0
\end{gathered}
$$

for any $Z \in D, \perp U$, where $U$ is unit vector orthogonal to $\xi, \alpha$ and $\mu$ are functions on $M$. M. Kimura [10] constructed ruled real hypersurfaces in complex projective space. Let $\bar{M}$ be a hypersurface in $S^{2 n+1}$ defined by

$$
\begin{gathered}
\left\{\left(r e^{i t} \cos \theta, r e^{i t} \sin \theta, \sqrt{1-r^{2}} z_{2}, \ldots, \sqrt{1-r^{2}} z_{n}\right) \in \mathbf{C}^{n+1} \mid\right. \\
\left.\sum_{j=2}^{n}\left|z_{j}\right|^{2}=1,0<r<1,0 \leq t, \theta<2 \pi,\right\} .
\end{gathered}
$$

Then the Hopf image $M$ of $\bar{M}$ is a minimal ruled hypersurface in $\mathbf{C} P^{n}$. We note that the above example of a ruled real hypersurface is not complete. In a similar way, in [1] the authors gave a minimal ruled real hypersurfaces in complex hyperbolic space. For more details about ruled real hypersurfaces we may refer to [13].

From Proposition 2, together with the results in [12] (in case of $P_{n} \mathbf{C}$ ) and [15] (in case of $H_{n} \mathbf{C}$ ) we get easily

THeORem 4. Let $M$ be a real hypersurface of $\tilde{M}_{n}(c), c \neq 0$. Suppose that $M$ satisfies $\varphi A+A \varphi=2 k \varphi$ for some non-zero constant $k$. Then the $C R$-structure is pseudo-hermitian and strongly pseudo-convex. Furthermore we have the following:

(I) in the case $\tilde{M}_{n}(c)=P_{n} \mathbf{C}$ with the Fubini-Study metric of $c=4$, then $M$ is locally congruent to one of the following:

$\left(A_{1}\right)$ a geodesic hypersphere of radius $r$, where $0<r<\frac{\pi}{2}$,

(B) a tube of radius $r$ over a complex quadric $Q_{n-1}$, where $0<r<\frac{\pi}{4}$.

(II) in the case $\tilde{M}_{n}(c)=H_{n} \mathbf{C}$ with the Bergman metric of $c=-4$, then $M$ is locally congruent to one of the following:

$\left(A_{0}\right)$ a horosphere,

$\left(A_{1}\right)$ a geodesic hypersphere or a tube over a complex hyperbolic hyperplane $H_{n-1} \mathbf{C}$,

(B) a tube over a totally real hyperbolic space $H_{n} \mathbf{R}$. 
Remark 1. (1) Together with Proposition 1, we see that the almost contact metric structure on $M$ which appears in the above theorem is a contact metric structure only for the very special case determined by $k= \pm 1$, where \pm depends on the orientation. More precisely, with the help of the tables in [3] and [16], we see that the almost contact metric structures are contact metric only for a geodesic hypersphere of radius $\frac{\pi}{4}$ in $P_{n} \mathbf{C}$, for a horosphere in $H_{n} \mathbf{C}$. Hence for real hypersurfaces appearing in Theorem 4, except those just mentioned, they do not admit contact structure but their associated CR structures are pseudo-hermitian, strongly pseudo-convex and further the g.-Tanaka-Webster connection $\hat{\nabla}$ defined on them coincides with the Tanaka-Webster connection.

(2) From (2.6), it follows that Levi-flat hypersurface is Levi-parallel. Leaving the Levi-flat case aside, we find that real hypersurfaces stated in Theorem 4 are also Levi-parallel.

We prepare some more results which are needed to prove our Main Theorem.

THeOREM 5 ([9]). Let $M$ be a Hopf hypersurface of $P_{n} \mathbf{C}$. Then $M$ has constant principal curvatures if and only if $M$ is locally congruent to one of the following:

$\left(A_{1}\right)$ a geodesic hypersphere of radius $r$, where $0<r<\frac{\pi}{2}$,

$\left(A_{2}\right)$ a tube of radius $r$ over a totally geodesic $P_{k} \mathbf{C}(1 \leq k \leq n-2)$, where $0<r<\frac{\pi}{2}$,

(B) a tube of radius $r$ over a complex quadric $Q_{n-1}$, where $0<r<\frac{\pi}{4}$,

(C) a tube of radius $r$ over $P_{1} \mathbf{C} \times P_{(n-1) / 2} \mathbf{C}$, where $0<r<\frac{\pi}{4}$ and $n(\geq 5)$ is odd,

(D) a tube of radius $r$ over a complex Grassmann $G_{2,5} \mathbf{C}$, where $0<r<\frac{\pi}{4}$ and $n=9$,

(E) a tube of radius $r$ over a Hermitian symmetric space $S O(10) / U(5)$, where $0<r<\frac{\pi}{4}$ and $n=15$.

Theorem 6 ([3]). Let $M$ be a Hopf hypersurface of $H_{n} \mathbf{C}$. Then $M$ has constant principal curvatures if and only if $M$ is locally congruent to one of the following:

$\left(A_{0}\right)$ a horosphere,

$\left(A_{1}\right)$ a geodesic hypersphere or a tube over a complex hyperbolic hyperplane $H_{n-1} \mathbf{C}$,

$\left(A_{2}\right)$ a tube over a totally geodesic $H_{k} \mathbf{C}(1 \leq k \leq n-2)$,

(B) a tube over a totally real hyperbolic space $H_{n} \mathbf{R}$. 
THeOREM 7 ([11], [15]). Let $M$ be a Hopf hypersurface of a non-flat complex space form $\tilde{M}_{n}(c), c \neq 0$. Suppose that the shape operator $A$ is $\eta$-parallel (i.e., $\left.g\left(\left(\nabla_{X} A\right) Y, Z\right)=0\right)$ for any tangent vectors $X, Y$ and $Z$ which are orthogonal to $\left.\xi\right)$. Then we have the following.

(I) In case that $\tilde{M}_{n}(c)=P_{n} \mathbf{C}$, then $M$ is locally congruent to one of real hypersurfaces of type $\left(A_{1}\right),\left(A_{2}\right)$ and $(B)$;

(II) In case that $\tilde{M}_{n}(c)=H_{n} \mathbf{C}$, then $M$ is locally congruent to one of real hypersurfaces of type $\left(A_{0}\right),\left(A_{1}\right),\left(A_{2}\right)$ and $(B)$.

\section{Levi-parallel Hopf Hypersurfaces in a Complex Space Form}

In this section we shall prove our Main Theorem. Suppose that $M$ is a Levi-parallel Hopf hypersurface of a complex space form $\tilde{M}_{n}(c)$ with respect to g.-Tanaka-Webster connection. Then by using (2.5) and (2.6) we have

$$
g\left(\left(\varphi\left(\hat{\nabla}_{Z} A\right)+\left(\hat{\nabla}_{Z} A\right) \varphi\right) X, \varphi Y\right)=0
$$

for any vector fields $X, Y, Z$ orthogonal to $\xi$ on $M$. It follows easily that

$$
g\left(\left(\hat{\nabla}_{Z} A\right) X, Y\right)-\eta\left(\left(\hat{\nabla}_{Z} A\right) X\right) \eta(Y)+g\left(\left(\hat{\nabla}_{Z} A\right) \varphi X, \varphi Y\right)=0
$$

for any $X, Y, Z \in D$.

Together with (2.4), we have

$$
\begin{aligned}
& g\left(\left(\nabla_{Z} A\right) X, Y\right)-\eta(A X) g(\varphi A Z, Y)-g(\varphi A Z, X) \eta(A Y) \\
& \quad+g\left(\left(\nabla_{Z} A\right) \varphi X, \varphi Y\right)-\eta(A \varphi X) g(\varphi A Z, \varphi Y)-g(\varphi A Z, \varphi X) \eta(A \varphi Y)=0
\end{aligned}
$$

for any $X, Y, Z \in D$. We now suppose that $A \xi=\alpha \xi$. Then (4.1) reduces to

$$
g\left(\left(\nabla_{Z} A\right) X, Y\right)-g\left(\varphi\left(\nabla_{Z} A\right) \varphi X, Y\right)=0
$$

where $X, Y, Z \in D$. Assume $X \in V_{\lambda}$, that is, $A X=\lambda X$, where we denote by $V_{\lambda}$ the eigenspace of $A$ associated with a principal curvature $\lambda$. Taking account of (3.3), we divide our arguments into two cases: (i) $2 \lambda \neq \alpha$ and $2 \lambda=\alpha$. First, we consider the case (i). Then for any $Z \in D$, we get

$$
\begin{aligned}
\left(\nabla_{Z} A\right) X & =\nabla_{Z}(A X)-A\left(\nabla_{Z} X\right) \\
& =(Z \lambda) X+(\lambda I-A)\left(\nabla_{Z} X\right) .
\end{aligned}
$$

So we have

$$
\begin{aligned}
g\left(\left(\nabla_{Z} A\right) X, X\right) & =Z \lambda+g\left((\lambda I-A) \nabla_{Z} X, X\right) \\
& =Z \lambda+g\left(\nabla_{Z} X,(\lambda I-A) X\right)=Z \lambda .
\end{aligned}
$$


Similarly, by using (3.3), we have

$$
g\left(\left(\nabla_{Z} A\right) \varphi X, \varphi X\right)=-(Z \lambda) \frac{\alpha^{2}+c}{(2 \lambda-\alpha)^{2}} .
$$

From (4.2), (4.3) and (4.4) we obtain

$$
(Z \lambda)\left(\lambda^{2}-\alpha \lambda-\frac{c}{4}\right)=0 .
$$

Since $\alpha$ is constant, this shows that

$$
Z \lambda=0 \text { for any } Z \in D \text {. }
$$

Also, it follows from the equation of Codazzi (3.2) that

$$
\left(\nabla_{Z} A\right) \xi-\left(\nabla_{\xi} A\right) Z=-\frac{c}{4} \varphi Z \text { for any } Z \in D .
$$

On the other hand, from (2.3) and (3.3) we find

$$
\begin{aligned}
\left(\nabla_{Z} A\right) \xi-\left(\nabla_{\xi} A\right) Z & =\nabla_{Z}(A \xi)-A \nabla_{Z} \xi-\nabla_{\xi}(A Z)+A\left(\nabla_{\xi} Z\right) \\
& =(\alpha I-A) \varphi A Z-(\xi \lambda) Z-(\lambda I-A) \nabla_{\xi} Z \\
& =\lambda\left(\alpha-\frac{\alpha \lambda+\frac{c}{2}}{2 \lambda-\alpha}\right) \varphi Z-(\xi \lambda) Z-(\lambda I-A) \nabla_{\xi} Z
\end{aligned}
$$

for any unit vector $Z \in V_{\lambda}$. From the above two equations, we obtain

$$
\xi \lambda=0
$$

where we have used $g(\varphi Z, Z)=0$ and $g\left((\lambda I-A) \nabla_{\xi} Z, Z\right)=0$. Hence from (4.5) and (4.6) we see that $\lambda$ is constant. Next, in the case (ii) $2 \lambda=\alpha$, since $\alpha_{1}$ is constant, $\lambda$ must be constant.

Thus, by virtue of Theorems 5 and 6 we can see that $M$ is locally congruent to one of six types $\left(A_{1}\right),\left(A_{2}\right),(B),(C),(D)$ and $(E)$ in $P_{n} \mathbf{C}$ or $\left(A_{0}\right),\left(A_{1}\right),\left(A_{2}\right)$ and $(B)$ in $H_{n} \mathbf{C}$. Conversely, by using Theorem 7 , we check that real hypersurfaces of types $\left(A_{1}\right),\left(A_{2}\right),(B)$ in $P_{n} \mathbf{C}$ or $\left(A_{0}\right),\left(A_{1}\right),\left(A_{2}\right)$ and $(B)$ in $H_{n} \mathbf{C}$ are Levi-parallel (with respect to the g.-Tanaka-Webster connection).

Now, we shall prove $M$ of types $(C),(D)$ and $(E)$ in $P_{n} \mathbf{C}$ is not Levi parallel. For $M$ of type $(C),(D)$ or $(E)$ in $P_{n} \mathbf{C}, M$ has five distinct constant principal curvatures, say $\lambda_{1}, \lambda_{2}, \lambda_{3}, \lambda_{4}$ and $\alpha$ so that $T M=V_{\lambda_{1}} \oplus V_{\lambda_{2}} \oplus V_{\lambda_{3}} \oplus$ $V_{\lambda_{4}} \oplus\{\xi\}_{\mathbf{R}}$. We put $x=\cot \left(\theta-\frac{\pi}{4}\right) \quad\left(\frac{\pi}{4}<\theta<\frac{\pi}{2}\right)$. Then we may express (cf. [11])

$$
\lambda_{1}=x, \quad \lambda_{2}=-\frac{1}{x}, \quad \lambda_{3}=\frac{x+1}{1-x}, \quad \lambda_{4}=\frac{x-1}{x+1}, \quad \alpha=\frac{-4 x}{x^{2}-1} .
$$


We note that

(4.8) $\quad 0<x<1 \quad$ and $\varphi V_{\lambda_{1}}=V_{\lambda_{2}}, \quad \varphi V_{\lambda_{2}}=V_{-\lambda_{1}}, \quad \varphi V_{\lambda_{a}}=V_{\lambda_{a}}, \quad a=3,4$.

We first prove the following

Lemma 1. Let $M$ be a real hypersurface $M$ of types $(C),(D)$ and $(E)$ in $P_{n}$ C. If $M$ is Levi-parallel, then

(1) for $X \in V_{\lambda_{i}}(i=1,2) ; \nabla_{Z} X=\left(\nabla_{Z} X\right)_{\lambda_{i}}-g(X, \varphi A Z) \xi$,

(2) for $X \in V_{\lambda_{a}}(a=3,4) ; \nabla_{Z} X=\left(\nabla_{Z} X\right)_{\lambda_{a}}-g(X, \varphi A Z) \xi$.

for any $Z \in D$, where $X_{\lambda}$ denotes the $V_{\lambda}$-component of the vector $X$.

Proof. For $X \in V_{\lambda}$ and $Y \in V_{\mu}$, we get

$$
g\left(\left(\nabla_{Z} A\right) X, Y\right)=(\lambda-\mu) g\left(\nabla_{Z} X, Y\right) .
$$

If we put $\bar{\lambda}=\frac{\alpha \lambda+2}{2 \lambda-\alpha}$, then $\varphi X \in V_{\bar{\lambda}}$ and $\varphi Y \in V_{\bar{\mu}}$. Together with (2.2) we get

$$
\begin{aligned}
g\left(\left(\nabla_{Z} A\right) \varphi X, \varphi Y\right) & =(\bar{\lambda}-\bar{\mu}) g\left(\nabla_{Z}(\varphi X), \varphi Y\right) \\
& =(\bar{\lambda}-\bar{\mu}) g\left(\varphi\left(\nabla_{Z} X\right), \varphi Y\right) \\
& =(\bar{\lambda}-\bar{\mu}) g\left(\nabla_{Z} X, Y\right)
\end{aligned}
$$

Suppose that $M$ is Levi-parallel. Then from (4.2) we obtain

$$
[(\lambda-\mu)+(\bar{\lambda}-\bar{\mu})] g\left(\nabla_{Z} X, Y\right)=0 .
$$

From (4.7) and (4.10) we calculate the following:

$$
\begin{aligned}
& \text { for } X \in V_{\lambda_{i}}(i=1,2), Y \in V_{\lambda_{3}} ; \frac{(x+1)\left(x^{2}+1\right)}{x(x-1)} g\left(\nabla_{Z} X, Y\right)=0, \\
& \text { for } X \in V_{\lambda_{i}}, Y \in V_{\lambda_{4}} ; \frac{(x-1)\left(x^{2}+1\right)}{x(x+1)} g\left(\nabla_{Z} X, Y\right)=0 \\
& \text { for } X \in V_{\lambda_{1}}, Y \in V_{\lambda_{2}} ; 2 x g\left(\nabla_{Z} X, Y\right)=0 \\
& \text { for } X \in V_{\lambda_{2}}, Y \in V_{\lambda_{1}} ;-2 x g\left(\nabla_{Z} X, Y\right)=0 \\
& \text { for } X \in V_{\lambda_{3}}, Y \in V_{\lambda_{4}} ; \frac{(x+1)\left(x^{2}+1\right)}{x(1-x)} g\left(\nabla_{Z} X, Y\right)=0 \\
& \text { for } X \in V_{\lambda_{4}}, Y \in V_{\lambda_{3}} ; \frac{(1-x)\left(x^{2}+1\right)}{x(x+1)} g\left(\nabla_{Z} X, Y\right)=0 .
\end{aligned}
$$


Since $g\left(\nabla_{Z} X, \xi\right)=-g(X, \varphi A Z)$, from (4.8) and (4.11), we may express $\nabla_{Z} X$ as (4.9).

Secondly, we also prove

Lemma 2. Let $M$ be a real hypersurface $M$ of type $(C),(D)$ and $(E)$ in $P_{n} \mathbf{C}$. Then we have

$$
\nabla_{\xi} Z \in V_{\lambda_{i}} \oplus\{\varphi Z\}_{\mathbf{R}} \quad \text { for } Z \in V_{\lambda_{i}}(i=1,2) .
$$

Proof. For any unit vector $Z \in V_{\lambda}$, from (2.3) and Proposition 3 it follows that

$$
\begin{aligned}
\left(\nabla_{Z} A\right) \xi-\left(\nabla_{\xi} A\right) Z & =\nabla_{Z}(A \xi)-A \nabla_{Z} \xi-\nabla_{\xi}(A Z)+A\left(\nabla_{\xi} Z\right) \\
& =(\alpha I-A) \varphi A Z-(\xi \lambda) Z-(\lambda I-A) \nabla_{\xi} Z \\
& =\lambda\left(\alpha-\frac{\alpha \lambda+2}{2 \lambda-\alpha}\right) \varphi Z-(\lambda I-A) \nabla_{\xi} Z .
\end{aligned}
$$

On the other hand, from (3.2) we get

$$
\left(\nabla_{Z} A\right) \xi-\left(\nabla_{\xi} A\right) Z=-\varphi Z .
$$

Hence we obtain

$$
(\lambda I-A) \nabla_{\xi} Z=\left[\lambda\left(\alpha-\frac{\alpha \lambda+2}{2 \lambda-\alpha}\right)\right] \varphi Z \quad \text { for } Z \in V_{\lambda} .
$$

Since $\varphi V_{\lambda_{1}}=V_{\lambda_{2}}$, from (4.13) we can find (4.12).

Thus, it follows from Proposition 3 and (4.13) that for $i=1,2$,

$$
\left\{\lambda_{i}-\frac{\alpha \lambda_{i}+2}{2 \lambda_{i}-\alpha}\right\} g\left(\nabla_{\xi} Z, \varphi Z\right)=\left[\lambda_{i}\left(\alpha-\frac{\alpha \lambda_{i}+2}{2 \lambda_{i}-\alpha}\right)\right] g(\varphi Z, \varphi Z)
$$

or

$$
2\left(\lambda_{i}^{2}-\alpha \lambda_{i}-1\right) g\left(\nabla_{\xi} Z, \varphi Z\right)=\alpha\left(\lambda_{i}^{2}-\alpha \lambda_{i}-1\right) g(\varphi Z, \varphi Z) .
$$

But, for a real hypersurface $M$ which is locally congruent to one of types $(C),(D)$ and $(E)$ we know that $\lambda^{2}-\alpha \lambda-1 \neq 0$. (We note that the equation $\lambda^{2}-\alpha \lambda-1=0$ holds if and only if $M$ is locally congruent to a real hypersurface of type $\left(A_{1}\right)$ or $\left(A_{2}\right)$.) Therefore from (4.14) we get 


$$
g\left(\nabla_{\xi} Z, \varphi Z\right)=\frac{\alpha}{2} g(\varphi Z, \varphi Z) \text { for } Z \in V_{\lambda_{i}}, i=1,2
$$

For $X \in V_{\lambda_{1}}$ and $Z \in V_{\lambda_{3}}$, by using (1) and (2) in (4.9), we have

$$
\begin{aligned}
R(Z, \varphi Z) X= & \nabla_{Z}\left(\nabla_{\varphi Z} X\right)-\nabla_{\varphi Z}\left(\nabla_{Z} X\right)-\nabla_{[Z, \varphi Z]} X \\
= & \nabla_{Z}\left\{\left(\nabla_{\varphi Z} X\right)_{\lambda_{1}}-\lambda_{3} g\left(X, \varphi^{2} Z\right) \xi\right\} \\
& -\nabla_{\varphi Z}\left\{\left(\nabla_{Z} X\right)_{\lambda_{1}}-\lambda_{3} g(X, \varphi Z) \xi\right\} \\
& -\nabla_{\left\{\left(\nabla_{Z} \varphi Z\right)_{\lambda_{3}}-\lambda_{3} \xi\right\}} X+\nabla_{\left\{\left(\nabla_{\varphi Z} Z\right)_{\lambda_{3}}+\lambda_{3} \xi\right\}} X \\
= & \left(\nabla_{Z}\left(\nabla_{\varphi Z} X\right)_{\lambda_{1}}\right)_{\lambda_{1}}-\lambda_{3} g\left(\left(\nabla_{\varphi Z} X\right)_{\lambda_{1}}, \varphi Z\right) \xi \\
& -\left(\nabla_{\varphi Z}\left(\nabla_{Z} X\right)_{\lambda_{1}}\right)_{\lambda_{1}}+\lambda_{3} g\left(\left(\nabla_{Z} X\right)_{\lambda_{1}}, \varphi^{2} Z\right) \xi \\
& -\left(\nabla_{\left(\nabla_{Z} \varphi Z\right)_{\lambda_{3}}} X\right)_{\lambda_{1}}+\lambda_{3} g\left(X, \varphi\left(\nabla_{Z} \varphi Z\right)_{\lambda_{3}}\right) \xi+\lambda_{3} \nabla_{\xi} X \\
& +\left(\nabla_{\left(\nabla_{\varphi Z} Z\right)_{\lambda_{3}}} X\right)_{\lambda_{1}}-\lambda_{3} g\left(X, \varphi\left(\nabla_{\varphi Z} Z\right)_{\lambda_{3}}\right) \xi+\lambda_{3} \nabla_{\xi} X .
\end{aligned}
$$

The equations (4.15) and (4.16) show that

$$
g(R(Z, \varphi Z) X, \varphi X)=2 \lambda_{3} g\left(\nabla_{\xi} X, \varphi X\right)=\alpha \lambda_{3} g(\varphi X, \varphi X) .
$$

On the other hand, since $\varphi X \in V_{\lambda_{2}}$ and $\varphi Z \in V_{\lambda_{3}}$, the equation of Gauss (3.1) gives

$$
g(R(Z, \varphi Z) X, \varphi X)=-2 g(\varphi Z, \varphi Z) g(\varphi X, \varphi X) .
$$

From this, together with (4.7), we have $\frac{-4 x}{x^{2}-1} \cdot \frac{1+x}{1-x}=-2$, that is, $x^{2}+1=0$. This is a contradiction.

Thus, we have our Main Theorem.

\section{References}

[ 1 ] S.-S. Ahn, S.-B. Lee and Y. J. Suh, COn ruled real hypersurfaces in a complex space form, Tsukuba J. Math. 17(2) (1993), 311-322.

[2] A. Bejancu, Geometry of CR-submanifolds, Mathematics and Its Application, D. Reidel Publ. Comp. (1986).

[ 3 ] J. Berndt, Real hypersurfaces with constant principal curvatures in complex hyperbolic space, J. Reine Angew. Math. 395 (1989), 132-141.

[4] D. E. Blair, Riemannian geometry of contact and symplectic manifolds, Progress in Mathematics, Birkäuser 203 (2001).

[5] A. Bogges, CR manifolds and the tangential Cauchy-Riemann complex, Press, Boca Raton (1991).

[6] T. E. Cecil and P. J. Ryan, Focal sets and real hypersurfaces in complex projective space, Trans. Amer. Math. Soc. 269 (1982), 481-499. 
[ 7 ] J. T. Cho, CR structures on real hypersurfaces of a complex space form, Publ. Math. Debrecen 54(3-4) (1999), 473-487.

[ 8 ] U.-H. Ki and Y. J. Suh, On real hypersurfaces of a complex space form, Math. J. Okayama 32 (1990), 207-221.

[9] M. Kimura, Real hypersurfaces and complex submanifolds in complex projective space, Trans. Amer. Math. Soc. 296 (1986), 137-149.

[10] M. Kimura, Sectional curvtures of holomorphic planes on a real hypersurface in $P^{n}(\mathbf{C})$, Math. Ann. 276 (1987), 487-497.

[11] M. Kimura and S. Maeda, On real hypersurfaces of a complex projective space, Math. Z. 202 (1989), 299-311.

[12] M. Kon, Pseudo-Einstein real hypersurfaces of complex space forms, J. Differential Geometry 14 (1979), 339-354.

[13] M. Lohnherr and H. Reckziegel, On ruled hypersurfaces in complex space forms, Kyungpook Math. J. 35(3) (1995).

[14] Y. T. Siu, Nonexistence of smooth Levi-flat hypersurfaces in complex projective spaces of dimensions $\geq 3$, Ann. of Math. (2) 151 (2000), 1217-1243.

[15] Y. J. Suh, On real hypersurfaces of a complex space form with $\eta$-parallel Ricci tensor, Tsukuba J. Math. 14 (1990), 27-37.

[16] R. Takagi, On homogeneous real hypersurfaces in a complex projective space, Osaka J. Math. 19 (1973), 495-506.

[17] R. Takagi, Real hypersurfaces in a complex projective space with constant principal curvatures I, II, J. Math. Soc. Japan 27 (1975); 43-53, 507-516.

[18] S. Tanno, Variational problems on contact Riemannian manifolds, Trans. Amer. Math. Soc. 314 (1989), 349-379.

[19] N. Tanaka, On non-degenerate real hypersurfaces, graded Lie algebras and Cartan connections, Japan J. Math. 2 (1976), 131-190.

[20] S. M. Webster, Pseudohermitian structures on a real hypersurface, J. Diff. Geometry 13 (1978), $25-41$.

Department of Mathematics

Chonnam National University

CNU The Institute of Basic Sciences

Kwangju 500-757, Korea

(Visiting Scholar in:)

University of Washington Mathematics Department

Box 354350 Seattle

WA 98195-4350, USA. 